\title{
Endopharyngeal Ultrasound
}

\author{
Taha Mur ${ }^{1}$, Osamu Sakai ${ }^{1}$, Lauren Tracy ${ }^{1}$, Kei Suzuki $^{1}$, and Daniel Faden ${ }^{2}$ \\ ${ }^{1}$ Boston Medical Center \\ ${ }^{2}$ Massachusetts Eye and Ear Infirmary
}

April 28, 2020

\begin{abstract}
Key Points: * We describe a novel procedure, Endopharyngeal Ultrasound (EPhUS) and EPhUS-guided FNA * EPhUS requires an operator and an assistant, can be performed transnasal or transoral, and utilizes a Endoscopic Ultrasonography Bronchoscope

* EPhUS is a safe and effective method for biopsy of deep space neck masses inaccessible to transcutaneous FNA
\end{abstract}

Key Points:

- We describe a novel procedure, Endopharyngeal Ultrasound (EPhUS) and EPhUS-guided FNA

- EPhUS requires an operator and an assistant, can be performed transnasal or transoral, and utilizes a Endoscopic Ultrasonography Bronchoscope

- EPhUS is a safe and effective method for biopsy of deep space neck masses inaccessible to transcutaneous FNA

Key Words: Endoscopic, Ultrasound, Fine needle aspiration, Neck mass, Biopsy, minimally invasive

Ethical Considerations: The patient presented below was informed that this procedure has not been reported in the past and agreed to proceed following standard informed consent. The patient consented to having their case published in the literature.

\section{INTRODUCTION:}

Ultrasound (US) has become an integral part of the practice of medicine due in part to its soft tissue resolution, cost effectiveness, ability to measure vessel flow and, perhaps most relevant to head and neck surgeons, ease of use in the clinical setting in conjunction with fine needle aspiration (FNA). Thus, it is no surprise that US has gained significant traction in head and neck surgery in both the clinical and operative setting for imaging and biopsies of soft tissue lesions of the lateral and central neck(2). The development of US probes attached to or passed through endoscopes has allowed for the development of endoscopic US. For instance, endobronchial ultrasound (EBUS) is a bronchoscopic technique that utilizes US to visualize structures in the trachea and paratracheal soft tissues and has become the modality of choice for mediastinal lymph node biopsy. In EBUS, the endoscope is equipped with an US at the tip, allowing for image guided endoscopic FNA. The EBUS system was first introduced in the 1990's as a minimally invasive option compared to mediastinoscopy and open biopsy. Large series have demonstrated the safety and cost effectiveness of EBUS with a major complication rate of well under 1\%(3).

Transcutaneous central and lateral neck US, in both the outpatient clinic and operating room, is commonplace in head and neck surgery. However, for the upper aerodigestive track, flexible endoscope-based US techniques and approaches have not yet been well developed or described. Adapting existing endoscopic based US techniques, here, we describe flexible endopharyngeal ultrasound (EPhUS) and the EPhUS guided FNA.

\section{TECHNICAL DESCRIPTION}


A 61-year-old woman was referred to the general Otolaryngology clinic with the incidental finding of a left neck mass identified on CT scan. Contrast-enhanced CT revealed a $3 \mathrm{~cm}$ low attenuating lesion with a punctate calcification, displacing the internal and external carotid arteries anteromedially and internal jugular vein posterolaterally(Figure 1A). The differential diagnosis included metastatic lymphadenopathy or neurogenic tumor. Given these findings, the patient was referred to interventional radiology for an image guided biopsy. However, upon review, the interventional radiology team deferred transcutaneous FNA given proximity of the mass to the great vessels. The patient was then referred to the head and neck surgical oncology clinic of the senior author for management. MRI with and without contrast demonstrated a rimenhancing, predominantly non-enhancing lesion with a nodular enhancement at its medial portion, and foci of susceptibility effect, suggestive of microhemorrhage(Figures 1B, C). Although, schwannoma was high on the differential diagnosis, calcifications were felt to be an unusual finding. The differential diagnosis included metastatic lesions, particularly from the thyroid, and neurogenic tumor. In office US confirmed that transcutaneous biopsy was not possible due to the location of the internal and external carotid arteries in the line of transcutaneous US-guided FNA. Open biopsy was felt to be a poor choice due to potential for nerve injury in the case of a neurogenic tumor, in addition to standard reasons to avoid open surgical biopsies in cases of suspected neoplasm (scar, risk of seeding tumor, cranial nerve injury). Options of observation and EPhUS were offered and after informed consent, EPhUS was selected.

The patient was given general anesthesia and intubated orally. A tonsil gag was placed to expose the pharynx. An Olympus BF-UC180F Endoscopic Ultrasonography Bronchoscope (Tokyo, Japan) was placed transorally into the pharynx(Figure 1D). The endoscope is equipped with a $7.5 \mathrm{MHz}$ convex ultrasound probe(Figure $1 \mathrm{E})$. The angle of view is 90 degrees and the direction of view is 35 degrees forward and oblique. The tip of this scope contains a balloon, which assists with acoustic coupling of the ultrasound, however this is of little utility in the pharynx. A working channel allows passage of a needle for FNA. The endoscope, processing tower and monitor system allow duel display of both the ultrasound and endoscopic images, including Doppler. Both transnasal and transoral EPhUS are possible. In our experience both require two people: an endoscopist and an assistant to hold the endoscope in contact with the pharyngeal wall. The endoscope is passed into the pharynx and then brought into contact with the pharyngeal mucosa. Real time ultrasonographic imaging is then performed. In this case example, the lesion in question, with the internal and external carotid arteries on the lateral surface, are clearly identifiable (Figure $1 \mathrm{~F}$ ). After confirmation of the lesion, FNA can be performed (Figure 1G).

This needle used here is a retractable, 22g-g beveled, needle with a stylet, which allows clearance of plugs. The external surface of the needle is grooved, rendering it more hyperechoic, improving US visualization. Once the target mass is identified, the needle is advanced through the channel of the bronchoscope. Although the needle can be extended to a length of $40 \mathrm{~mm}$, intrinsic safety mechanisms of the device prevent excessive needle protrusion. Suction can be applied with a 20-ml VacLoc syringe as needed. When an adequate biopsy is obtained, the entire needle system is then withdrawn and the aspirated specimen is removed by pushing preservative through the lumen. Both on-site and final pathology confirmed the diagnosis of nerve sheath tumor. There was no minimal blood loss. The patient was observed in the post-anesthesia unit and discharged in stable condition after examination confirmed no voice changes, dysphagia or shortness of breath. When seen in follow up, she was asymptomatic.

\section{DISCUSSION}

Endopharyngeal ultrasonographic techniques have been sparsely described in the

literature8,9,10. All such descriptions have employed rigid endocavitary probes, which

suffer from limitations in: 1) the anatomic regions accessible to line of sight through the oral

aperture and 2) cumbersome size. Here, we present EPhus and EPhUS-guided FNA as a safe and minimally invasive technique for assessing and sampling parapharyngeal lesions not accessible by transcutaneous FNA. While EPhUS requires specialized equipment, the procedure is straight forward for surgeons familiar with endoscopy of the upper aerodigestive tract and ultrasonography. Most hospitals which provide multispecialty 
care are equipped with the necessary endoscopes to perform EPhUS. In this case example, a lesion of the carotid space, was successfully visualized and sampled by EPhUS. Additional spaces, which have traditionally proved hard to access by transcutaneous image (US or CT) guided FNA, yet are accessible by EPhUS include the parapharyngeal, retropharyngeal and pterygomandibular spaces (Figures 2A-B).

In conclusion, EPhUS and EPhUS guided FNA is a safe, minimally invasive approach for visualization and biopsy of lesions in the parapharyngeal, retropharyngeal and pterygomandibular spaces, which have traditionally been difficult to access with transcutaneous image guided FNA.

\section{REFERENCES :}

1. Kane D, Grassi W, Sturrock R, Balint PV. A brief history of musculoskeletal ultrasound:'From bats and ships to babies and hips'. Rheumatology. 2004 Jul 1;43(7):931-3.

2. Mcivor NP, Freeman JL, Salem S, Elden L, Noyek AM, Bedard YC. Ultrasonography and ultrasoundguided fine-needle aspiration biopsy of head and neck lesions: a surgical perspective. The Laryngoscope. 1994 Jun;104(6):669-74.

3. Rintoul RC, Glover MJ, Jackson C, Hughes V, Tournoy KG, Dooms C, Annema JT, Sharples LD. Cost effectiveness of endosonography versus surgical staging in potentially resectable lung cancer: a health economics analysis of the ASTER trial from a European perspective. Thorax. 2014 Jul 1;69(7):679-81.

4. Gu P, Zhao YZ, Jiang LY, Zhang W, Xin Y, Han BH. Endobronchial ultrasound-guided transbronchial needle aspiration for staging of lung cancer: a systematic review and meta-analysis. European journal of cancer. 2009 May 1;45(8):1389-96.

5. Steinfort DP, Hew MJ, Irving LB. Bronchoscopic evaluation of the mediastinum using endobronchial ultrasound: a description of the first 216 cases carried out at an Australian tertiary hospital. Internal medicine journal. 2011 Dec;41(12):815-24.

6. Fornage BD, Edeiken BS, Clayman GL. Use of transoral sonography with an endocavitary transducer in diagnosis, fine-needle aspiration biopsy, and intraoperative localization of retropharyngeal masses. American Journal of Roentgenology. 2014 May;202(5):W481-6.

Figure legends

Figure 1. A 61-year-old woman with an incidentally found left neck mass.

1. Contrast-enhanced CT demonstrates a well-demarcated lesion (arrows ) with a punctate calcification (arrowhead) displacing the internal and external carotid arteries anteromedially and internal jugular vein posterolaterally. Note the lesion is surrounded by great vessels, preventing percutaneous biopsy.

2. Post-contrast fat-suppressed T1-weighted MR image demonstrates a rim-enhancing, predominantly non-enhancing lesion (arrows) with a nodular enhancement (arrowhead) at its medial portion, which may be an ideal site for biopsy.

3. Fast-field echo MR image demonstrates foci of susceptibility effect (arrowheads) within the lesion (arrows), suggestive of microhemorrhage.

4. A Crow-Davis is used to expose the pharynx. An assistant works to keep the bronchoscope in position.

5. The endoscope is equipped with a $7.5 \mathrm{MHz}$ convex ultrasound probe and a needle that is delivered through the working channel of the scope.

6. Endoscopic Doppler ultrasonography demonstrates the lesion (arrows) and surrounding internal and external carotid vessels (arrowheads).

7. Intra-procedure ultrasonography demonstrates needle (arrows) inserted into the lesion for sampling.

Figure 2. Examples of lesions that may be accessed by EPhUS guided FNA

1. A 53-year-old man with laryngeal cancer. Contrast-enhanced CT demonstrates a borderline-enlarged round retropharyngeal node with ill-defined boundary (arrow ).

2. A 73-year-old woman with mandibular cancer status post resection and reconstruction. Contrastenhanced CT demonstrates a peripherally enhancing lesion (arrow) at the medial and posterior aspect of the right medial pterygoid muscle, concerning for recurrence. 


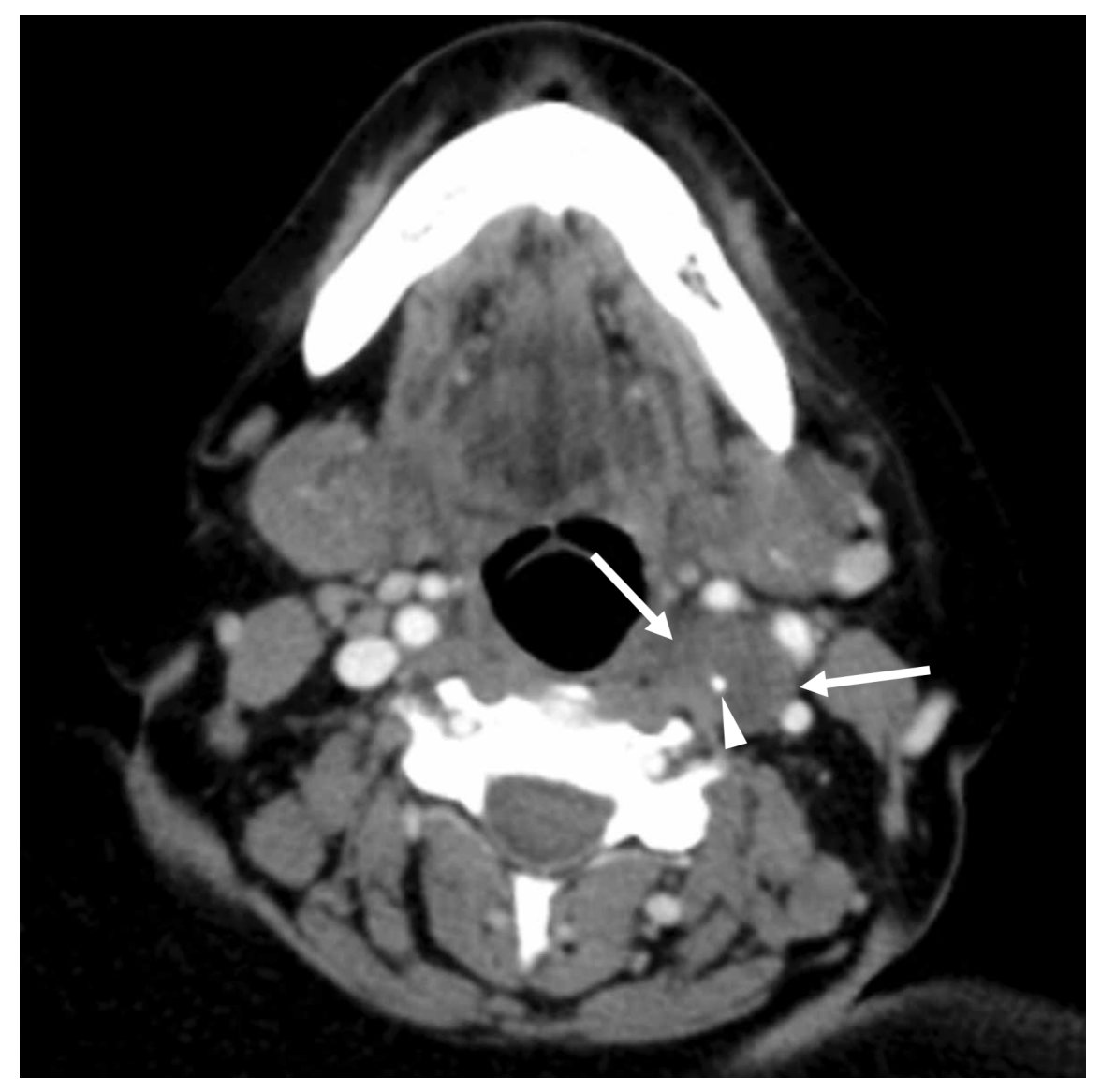




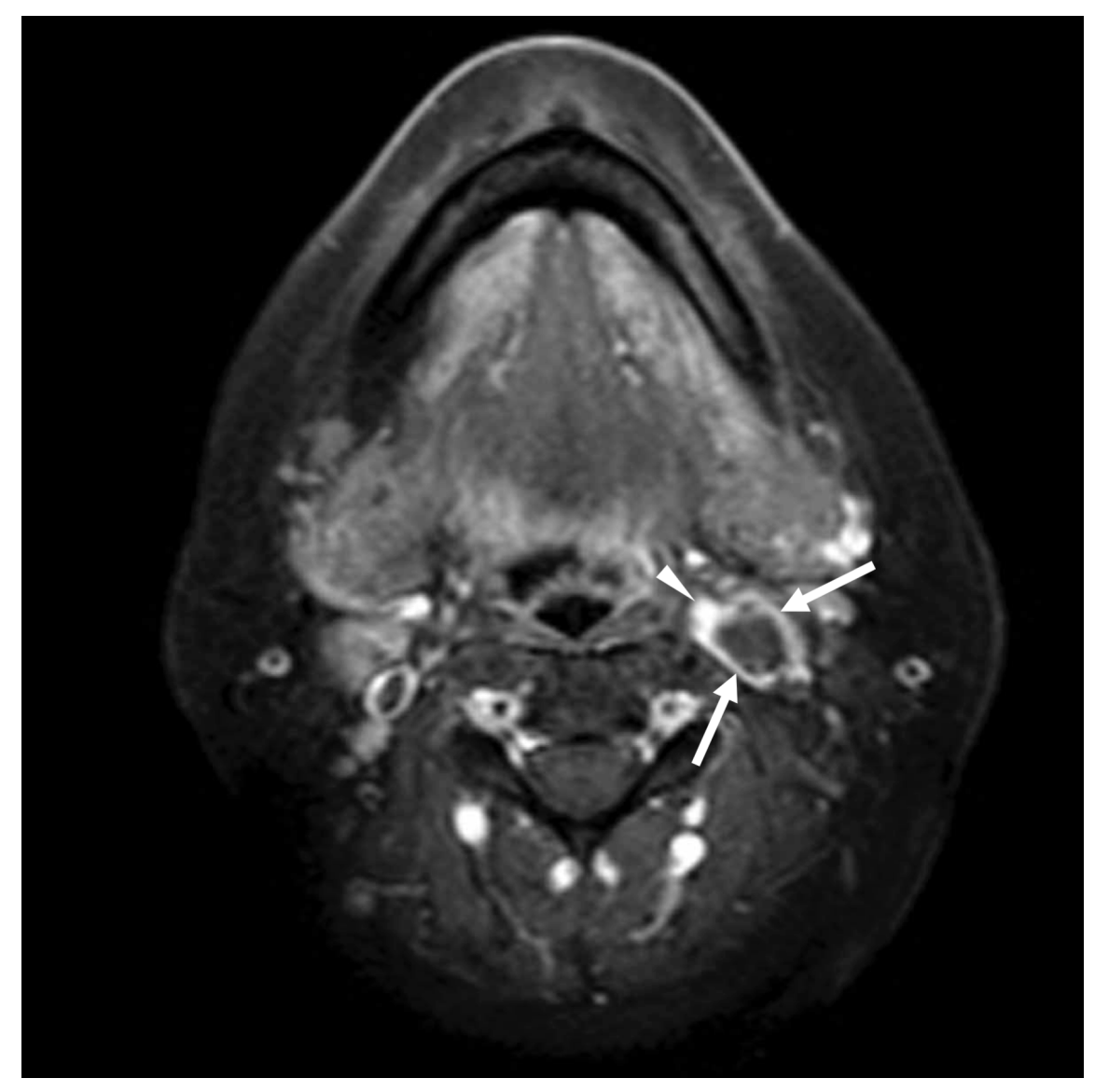




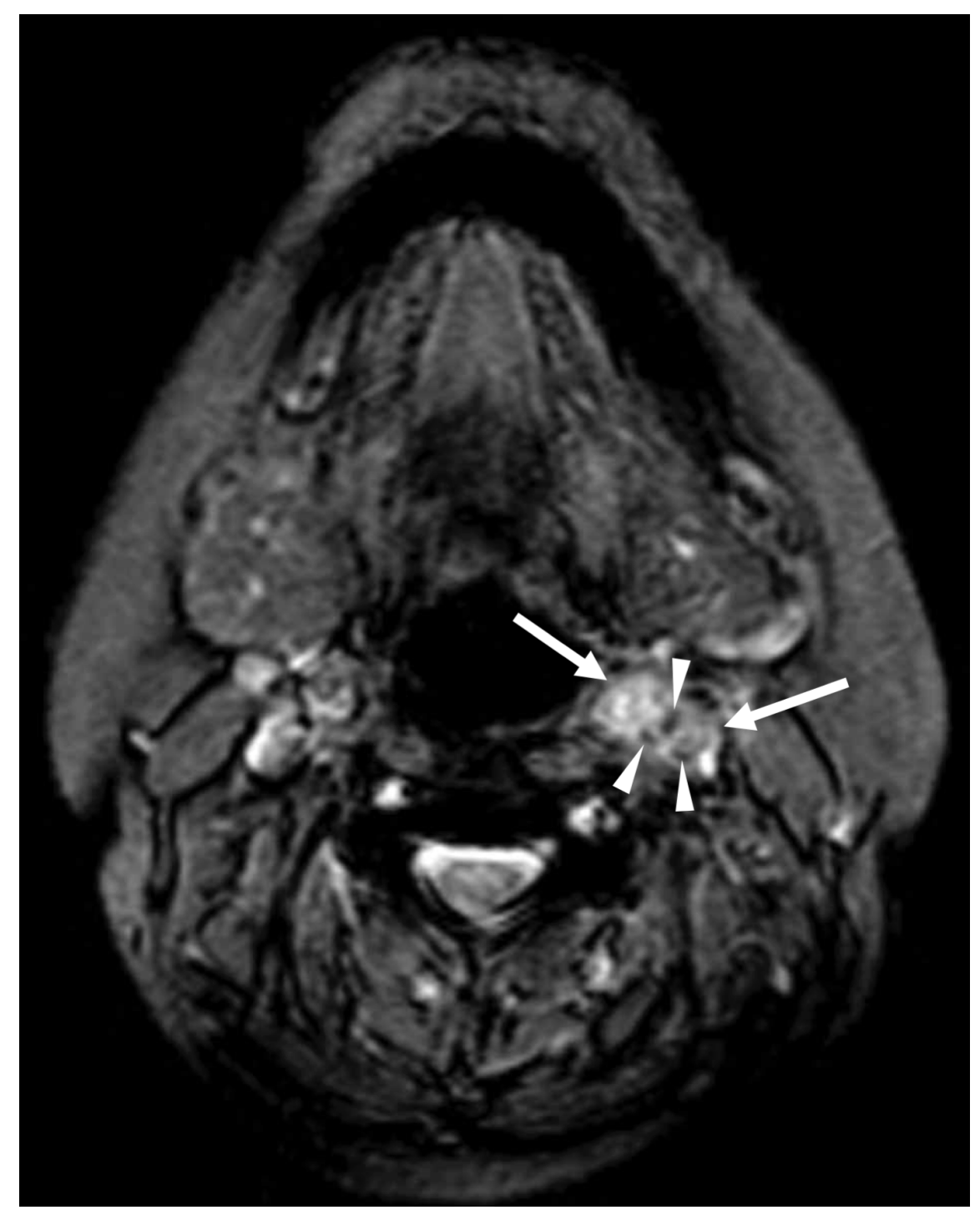




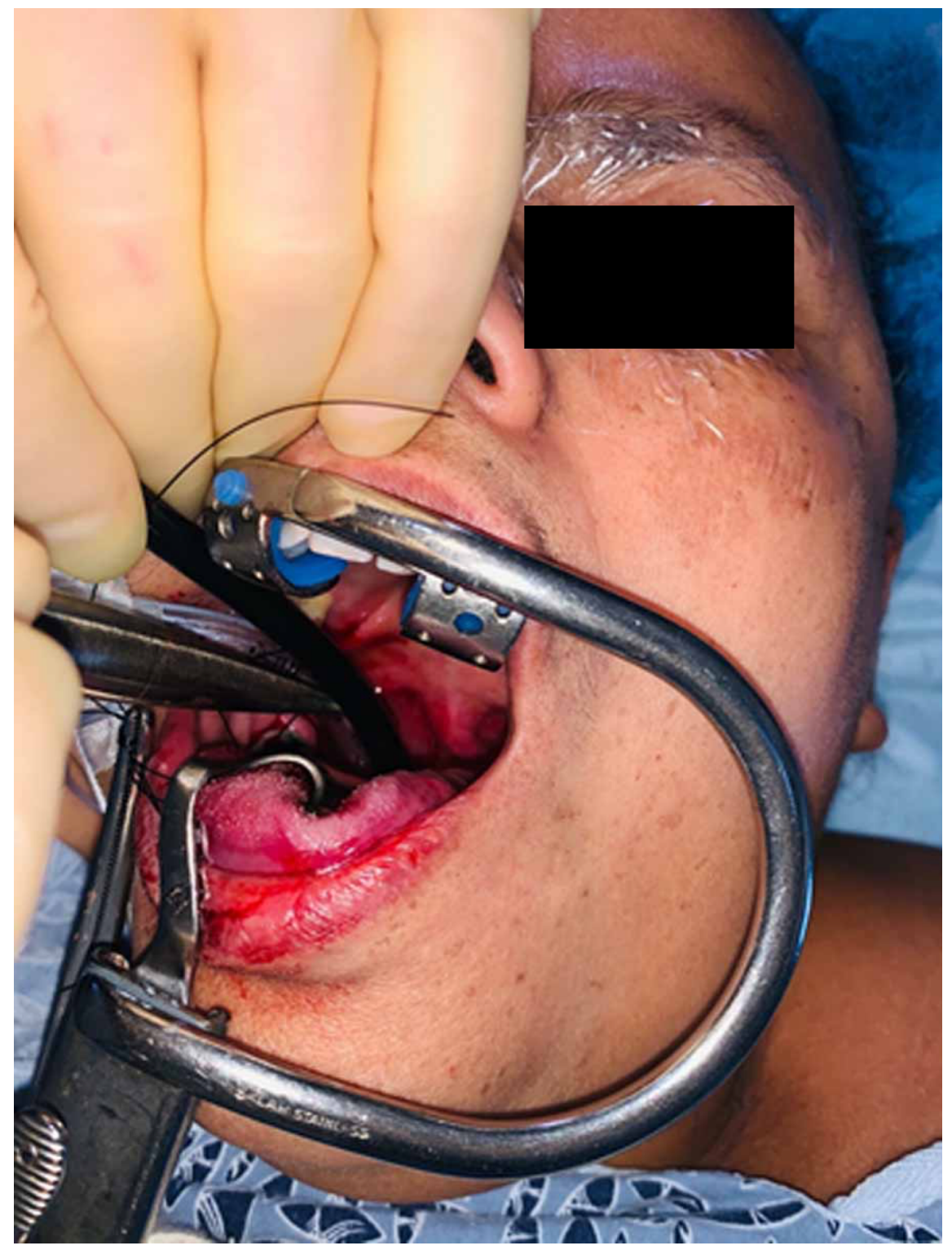



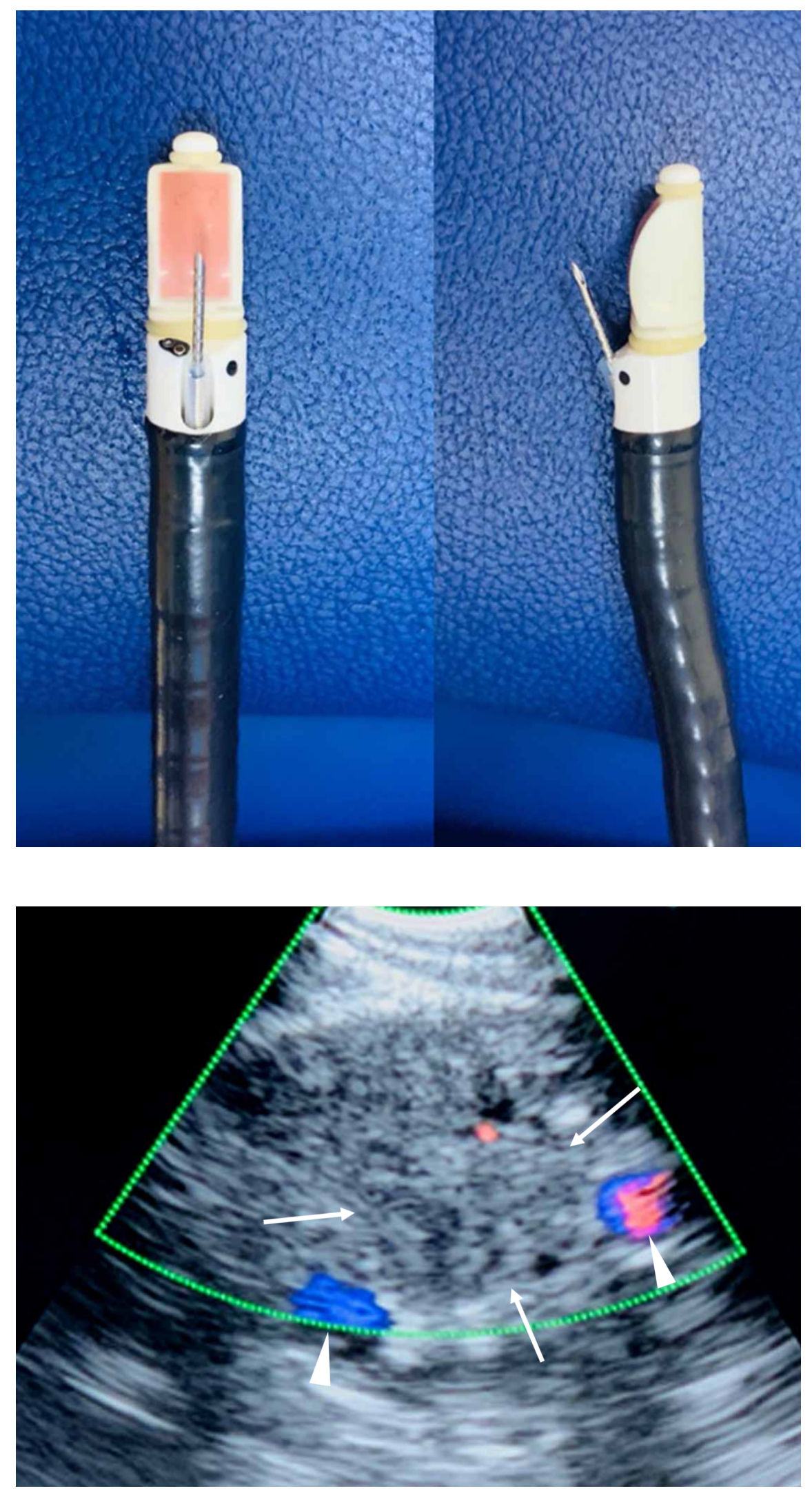


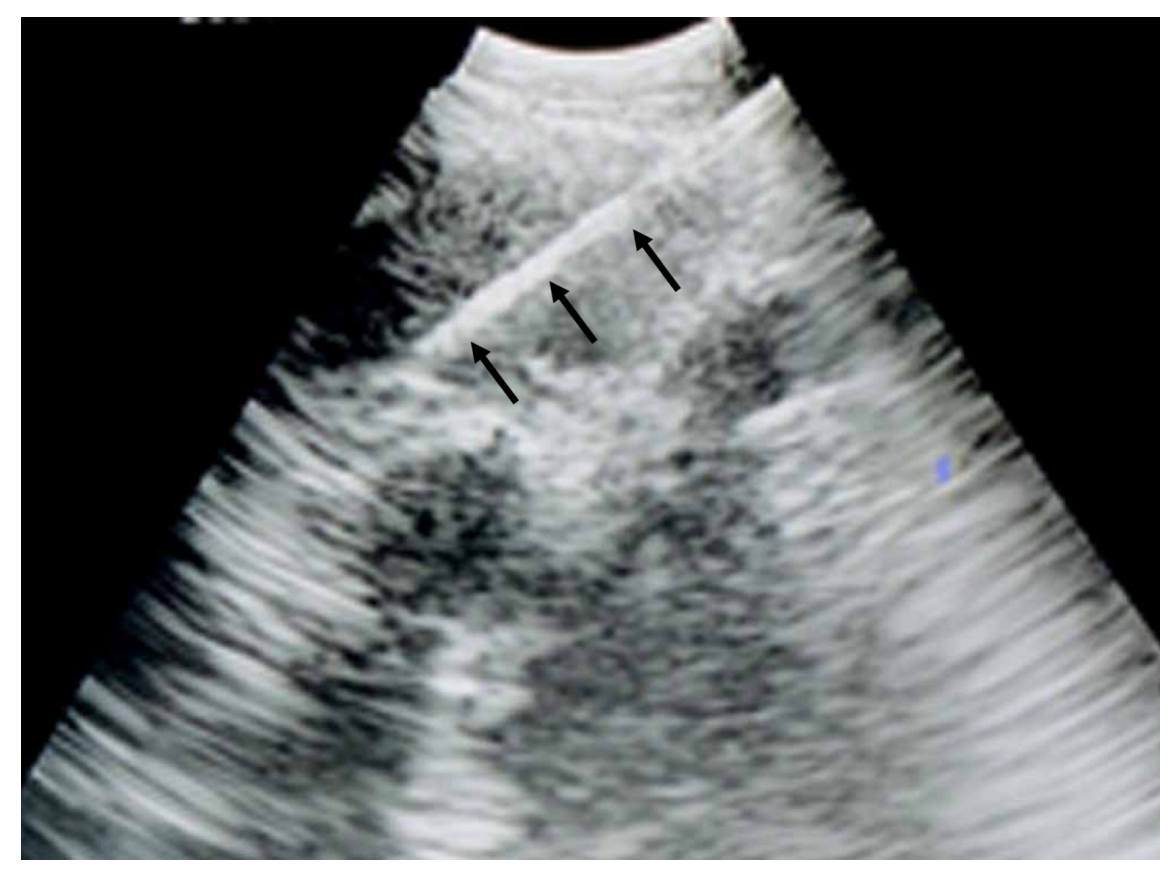




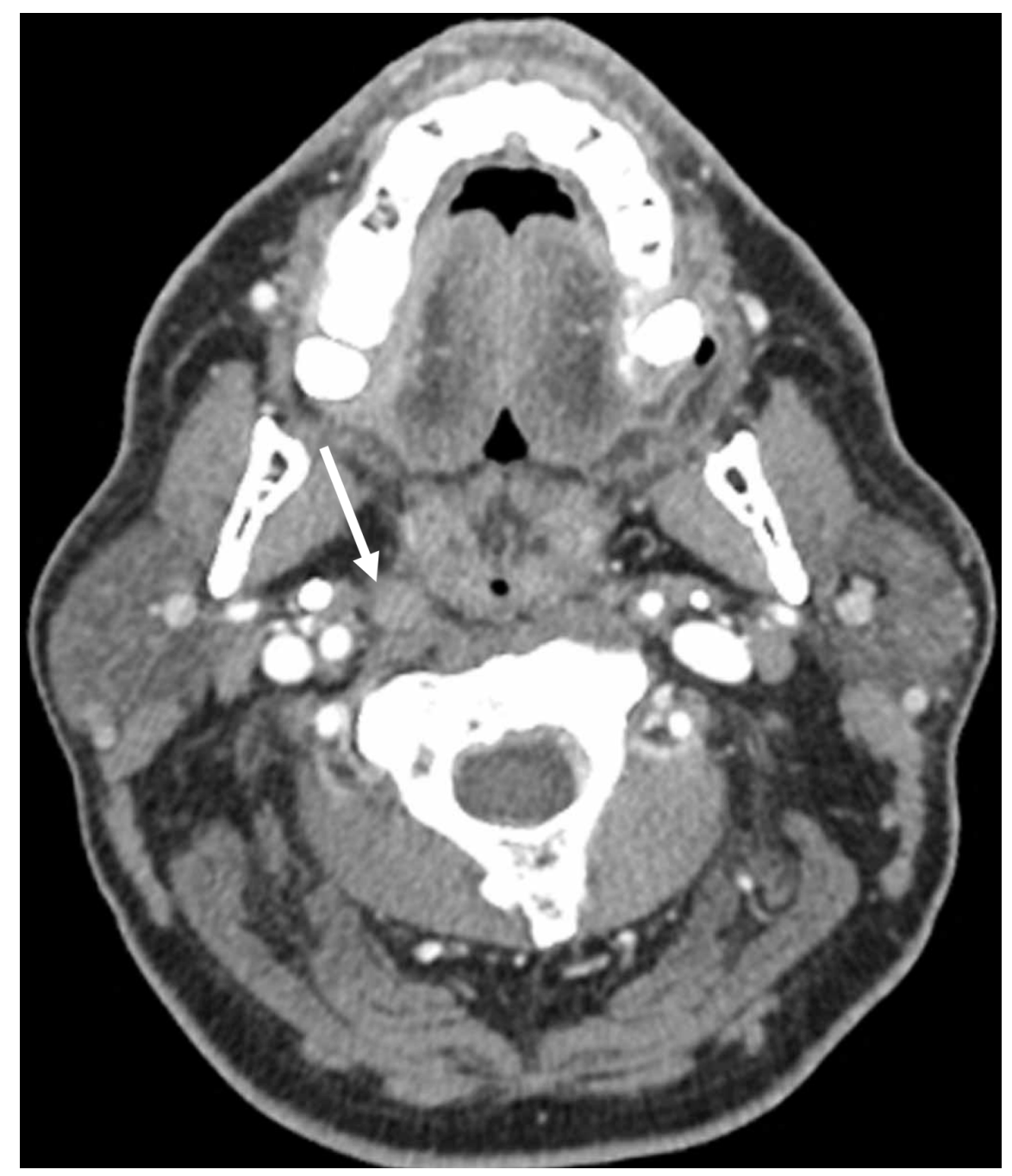




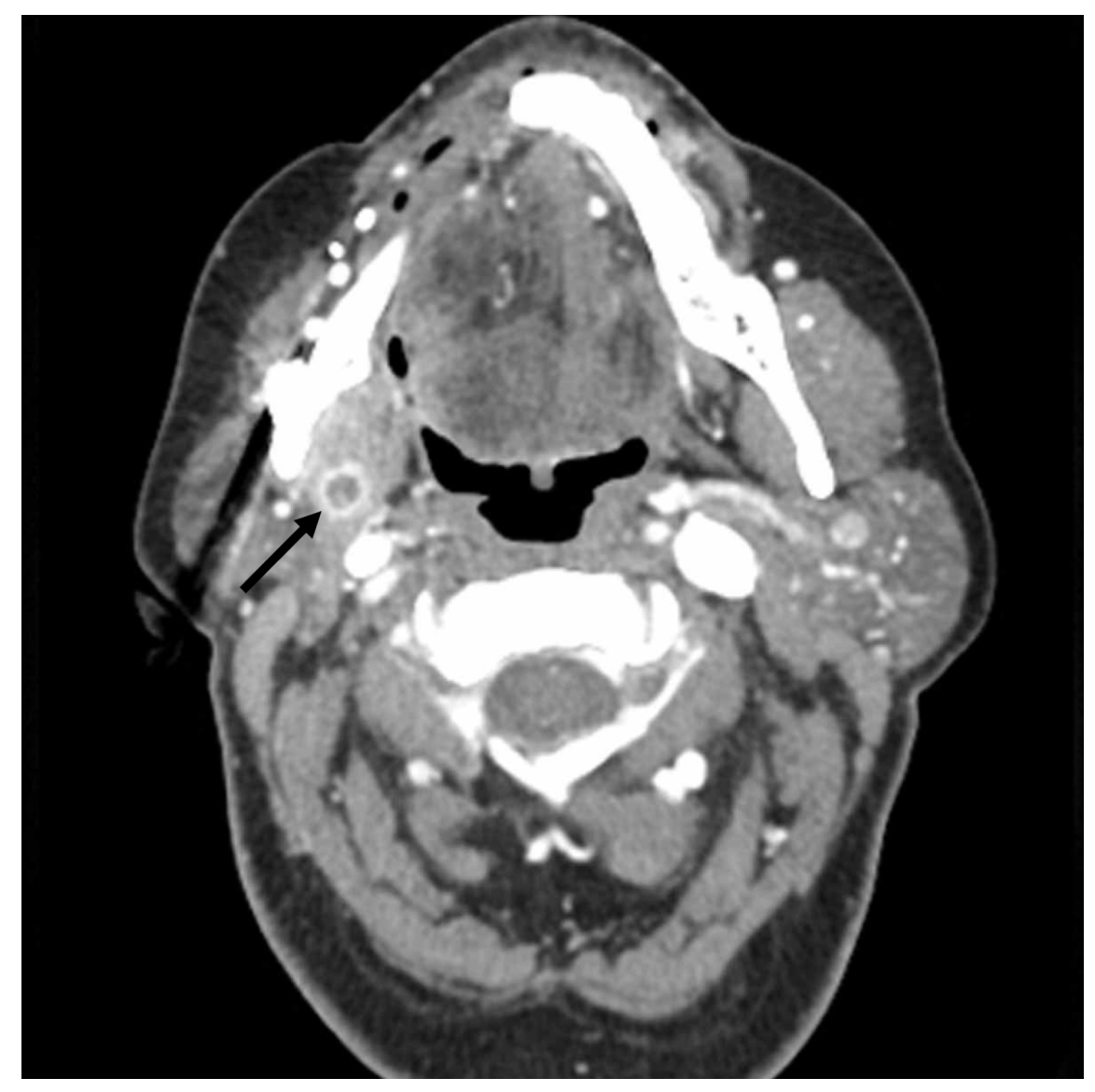




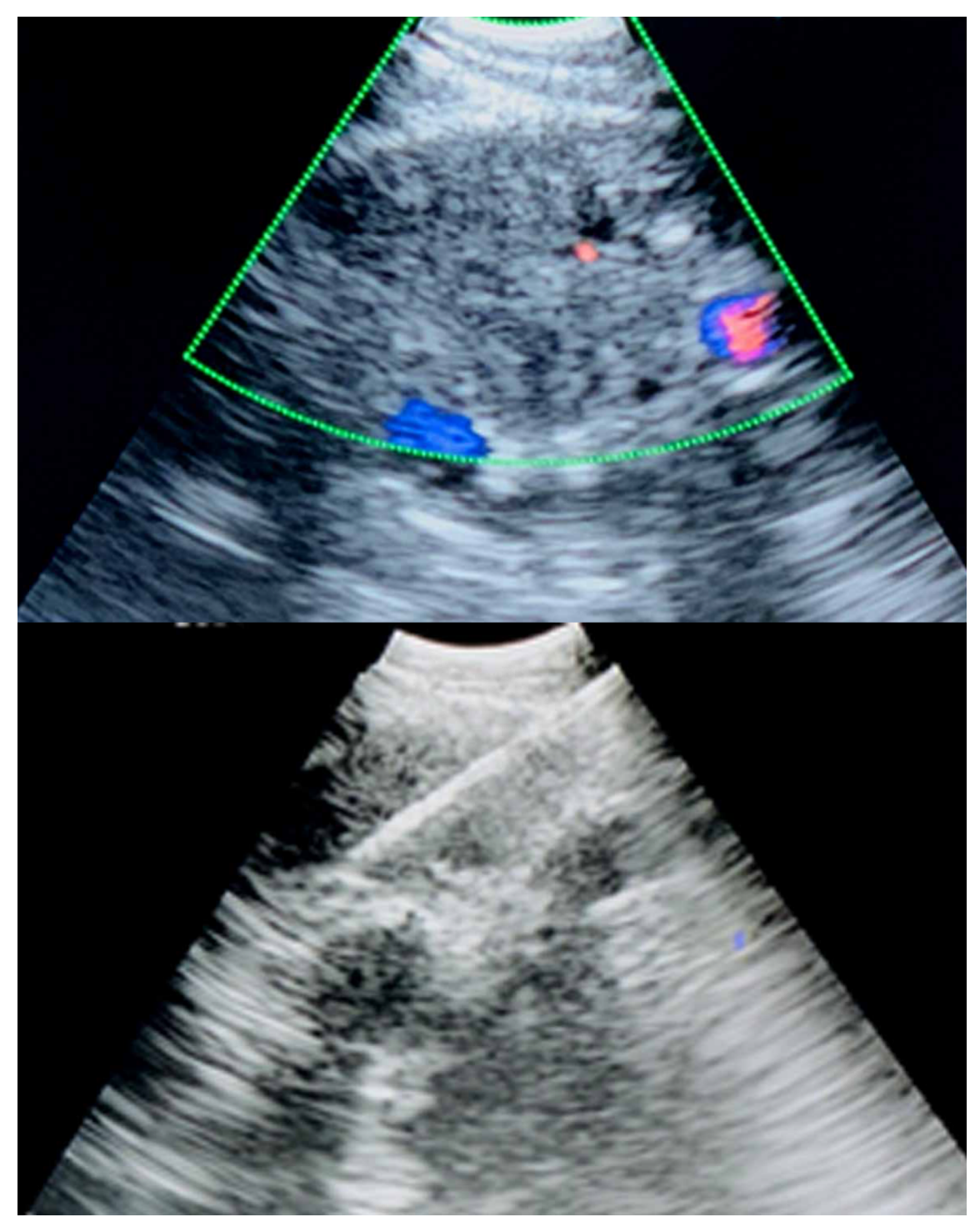




\section{CONSENT AND RELEASE}

I, Ana Santing d am being treated by Dr. Daniel Faden in the Department of Otolaryhgology at Boston Medical Center. Dr. Faden will be performing facial reconstructive surgery on me. In the course of my treatment, Dr. Faden will be taking preoperative and postoperative photographs of me to document the change in my appearance. Dr. Faden may also take intraoperative photographs of my procedures. These photographs will be considered as part of my medical record and will be maintained by Boston Medical Center as required by law.

Dr. Faden has requested my consent to his use of these photographs for educational and scientific purposes, including lectures and presentations for medical students, residents and other health care providers, as well as inclusion in journal articles and books.

He would also like to use them for marketing purposes. Marketing could include showing my photographs to health care providers who might refer patients to Dr. Faden for care or placing my photographs in a brochure or on a website. Dr. Faden would also like to show these photographs to prospective patients who are considering a similar type of surgery.

When Dr. Faden uses the photographs. for educational, scientific or marketing purposes, he may also disclose my health history, complaints and diagnoses (health information).

Dr. Faden has told me that he will not use my name when he uses these photographs. However, I understand that my face may be identifiable to persons who see the photographs. In giving my consent, I understand that I am giving up my right to privacy and confidentiality concerning the use of my face and the disclosure of health information which I might otherwise have had. I have initialed the type of disclosure of photographs and health information which I hereby
authorize.

AS Educational or scientific purposes

I understand that I will receive no financial compensation for the use of these photographs or the disclosure of my health information.

I also understand that my consent to the use of these photographs and the disclosure of my health information is completely voluntary. Had I declined to consent, such refusal would have no effect on the availability or quality of care which I would receive from either Dr.

Faden or Boston Medical Center.

I have been informed that I may take back my consent to use these photographs and my health information by writing to:

Boston Medical Center Privacy Officer

Boston Medical Center

One Boston Medical Center Place

Boston, Massachusetts 02118 
If I take back my consent, it will not affect any disclosures made by Dr. Faden prior to the receipt of my letter.

My authorization to use my photographs and health information will expire if I contact the hospital's privacy officer. I have received a copy of this Consent and Release.

I have read and understand the information contained in this consent and release form. All my questions have been answered to my satisfaction.

I consent to the use of my photographs and disclosure of my health information as described above.

Print Name: Ana SAntiago

signature:t Ana SAnty

Date/Time: 1/16/2020 11:AM

Witness:

Witness: $1 / 16 / 2020$ llies $A \mu$

